\title{
Funciones neurocognitivas en estudiantes del sexto semestre de la Escuela de Ciencias Psicológicas
}

\section{Neurocognitive functions in students of the sixth semester of the School of Psychological Sciences}

Recibido 07/04/2021

Mariela Dayane Chavarría Pérez

Maestría en Docencia Universitaria

Universidad de San Carlos de Guatemala

dayane599@yahoo.com.mx

https://orcid.org/0000-0001-7234-7415

Aceptado 02/07/2021

\section{Referencia}

Chavarría Pérez, M. D. (2021). Funciones neurocognitivas en estudiantes del sexto semestre de la Escuela de Ciencias Psicológicas. Revista Docencia Universitaria, 2(2), 52-59.

https://doi.org/10.46954/revistadusac.v2i2.31

\section{Resumen}

Este artículo tiene como objetivo presentar los resultados de la investigación respecto a las "Funciones neurocognitivas presentes en estudiantes del sexto semestre de la carrera técnica de Terapia Ocupacional y Recreativa, de la Escuela de Ciencias Psicológicas", identificar cuál tiene incidencia en los procesos superiores de aprendizaje, determinar cuales se desarrollan en el proceso de aprendizaje y finalmente los diferentes procesos que se ejecutan. El motivo principal es detectar cada proceso neurocognitivo y así desarrollar los cursos de manera efectiva. La investigación fue de enfoque cualitativo, alcance exploratorio y diseño fenomenológico. La muestra fue a conveniencia con un grupo de 22 estudiantes hombre y mujeres en edades de los 18 a 35 años. En los resultados finales se identificó que las funciones neurocognitivas evaluadas el 
conocimiento declarativo y condicional, se van desarrollando a lo largo de los procesos educativos con mayor diferencia que el declarativo y finalmente se concluye que en la realización del análisis de los procesos neurocognitivos, la planificación y la anticipación, son las que brindan una alta frecuencia en el proceso de educación superior.

\section{Abstract}

This article aims to present the results of the research regarding the "Neurocognitive functions present in students of the sixth semester of the technical career of Occupational and Recreational Therapy, of the School of Psychological Sciences", to identify which has an impact on the higher processes of learning, determine which are developed in the learning process and finally the different processes that are executed. The main reason is to detect each neurocognitive process and thus develop the courses effectively. The research was of a qualitative approach, exploratory scope, and phenomenological design. The sample was at convenience with a group of 22 male and female students aged 18 to 35 years. In the final results, it was identified that the neurocognitive functions evaluated, declarative and conditional knowledge, develop throughout the educational processes with a greater difference than the declarative one and finally it is concluded that in the analysis of neurocognitive processes, planning and anticipation, are those that provide a high frequency in the higher education process.

\section{Introducción}

Este artículo aborda el tema del desarrollo académico de la educación superior, se investigó y acorde a Mayer (2006) la importancia de los procesos neurocognitivos involucrados al estudiar de manera sistemática, organizada, el recorrido académico, se ha de tener claridad que de acuerdo con cada sujeto en su individualidad tiene una forma de adquirir y llevar a la práctica todos aquellos conocimientos.

Dentro de la Escuela de Ciencias Psicológicas se detectan funciones neurocognitivas que presenta el grupo de estudiantes. Las funciones neurocognitivas que se dividen en 8: orientación, atención, concentración, codificación, lenguaje, lectura, escritura, funciones ejecutivas y de evocación, (Mayer, 2006) utilizadas por los estudiantes
Palabras clave:

diseño fenomenológico, funciones neurocognitivas, investigación cualitativa, procesos de aprendizaje
Keywords: phenomenological design, neurocognitive functions, qualitative research, learning processes 
en el nivel de educación superior, las que implementan al momento de realizar las actividades académicas, acciones que requieran hacer uso de las funciones neurocognitivas, se debe reconocer que estas se potencializan o tendrán mayor habilidad en alguna de ellas de acuerdo a la utilidad que se les dé (Bonilla, 2017).

En la investigación cualitativa suelen producirse con mucha frecuencia preguntas antes, durante y después de la recolección y el análisis de datos, pero también es conveniente para comprender tales fenómenos, buscar patrones, experiencias y sobre todos los significados de cada uno, predomina el razonamiento inductivo, que explora y describe, donde el proceso de indagación es flexible, es interpretativo. El alcance de esta investigación es exploratorio ya que está dirigida a investigar un fenómeno, identificará algunos conceptos para estudios más amplios y desde una perspectiva innovadora. El diseño fenomenológico explora, describe y comprende todas las experiencias de la persona y como se comparte la experiencia (Hernández \& Mendoza, 2018 ).

\section{Materiales y métodos}

La investigación es cualitativa que hace referencia a la naturaleza, carácter y propiedades de los fenómenos. La muestra de estudio fue conformada por un grupo de 22 estudiantes, 4 hombres y 18 mujeres, de edades entre 18 a 35 años; de la Escuela de Ciencias Psicológicas del VI semestre de la carrera técnica de Terapia Ocupacional y Recreativa. Se utilizaron dos instrumentos para recabar la información: el Inventario de Habilidades Metacognitivas -MAl- y el cuestionario sobre funciones ejecutivas.

Las funciones neurocognitivas son aquellas capacidades y aptitudes del ser humano relacionadas con el procesamiento de información como lo son la memoria, la atención, la percepción, la creatividad, el pensamiento abstracto, el almacenamiento, las respuestas, todas ellas experiencias para desarrollar tareas más complejas, entre otras (Rios et al. , 2017).

Entre los hallazgos más importantes que se pueden incluir, están el cuestionario sobre funciones ejecutivas sobre: la planificación y la anticipación que son funciones que realizan con mayor incidencia en sus actividades académicas; la fijación de metas, estimación temporal, 
el razonamiento, la toma de decisiones y las multitareas, obtuvieron un segundo dato alto, la inhibición que es la que regula la conducta ocupa el tercer lugar los datos; la ejecución dual, la monitorización, la memoria de trabajo verbal y no verbal así como la organización son ubicadas en el último plano de sus actividades académicas.

Según, Díaz y Hernández mantener un estado mental propicio para el aprendizaje es una condición que incluye el fortalecimiento de los elementos motivacionales y la concentración. Barriga (2010) están asociadas a los recursos del estudiante y a los cuatro tipos de conocimiento: 1. Los conocimientos cognitivos básicos, 2. La base de los conocimientos, 3 . El conocimiento estratégico y 4 . El conocimiento meta-cognitivo (Páez, 2006).

El otro instrumento utilizado, el inventario de habilidades neurocognitivas -MAl- el conocimiento declarativo que se refiere a la información que tiene el estudiante de sí mismo y la conciencia de los factores que afectan el aprendizaje, sus habilidades y el uso de capacidades cognitivas; el conocimiento procedimental que es la ejecución de las habilidades, el empleo de estrategias, recursos en el proceso; el conocimiento procedimental se refiere a cuándo y porqué utilizará estrategias de aprendizaje, la planificación que es el establecimiento de metas y la selección de los recursos, la organización que es gestionar la información del aprendizaje, el monitoreo es la conciencia de comprender la tarea y ejecutarla, la depuración son acciones que se realizan para corregir errores durante el desarrollo de la tarea y ajustar las estrategias para un mejor aprendizaje y finalmente la evaluación que son los juicios de valor y el análisis de los aprendizajes logrados que les permiten ir evaluando la efectividad de las estrategias de aprendizaje utilizadas.

El proceso de metacognición consiste en orientar a los estudiantes a la reflexión sobre su desempeño y se autorregulen, que sean capaces de mejorar su aprendizaje y resuelvan los problemas académicos con todos los recursos personales que posean, con el fin de comprender lo que se va a hacer y tomar conciencia de cómo actuar de la mejor manera posible, para alcanzar los logros e identificarlos. (Tobon, et al., 2010) 


\section{Resultados}

Entre los hallazgos encontrados, se puede mencionar que los datos brindados reflejan de forma clara que los estudiantes utilizan sus funciones neurocognitivas en las actividades académicas en su formación académica en educación superior.

Figura 1. Resultados de la categoría de conocimiento declarativo

\section{CONOCIMIENTO DECLARATIVO}

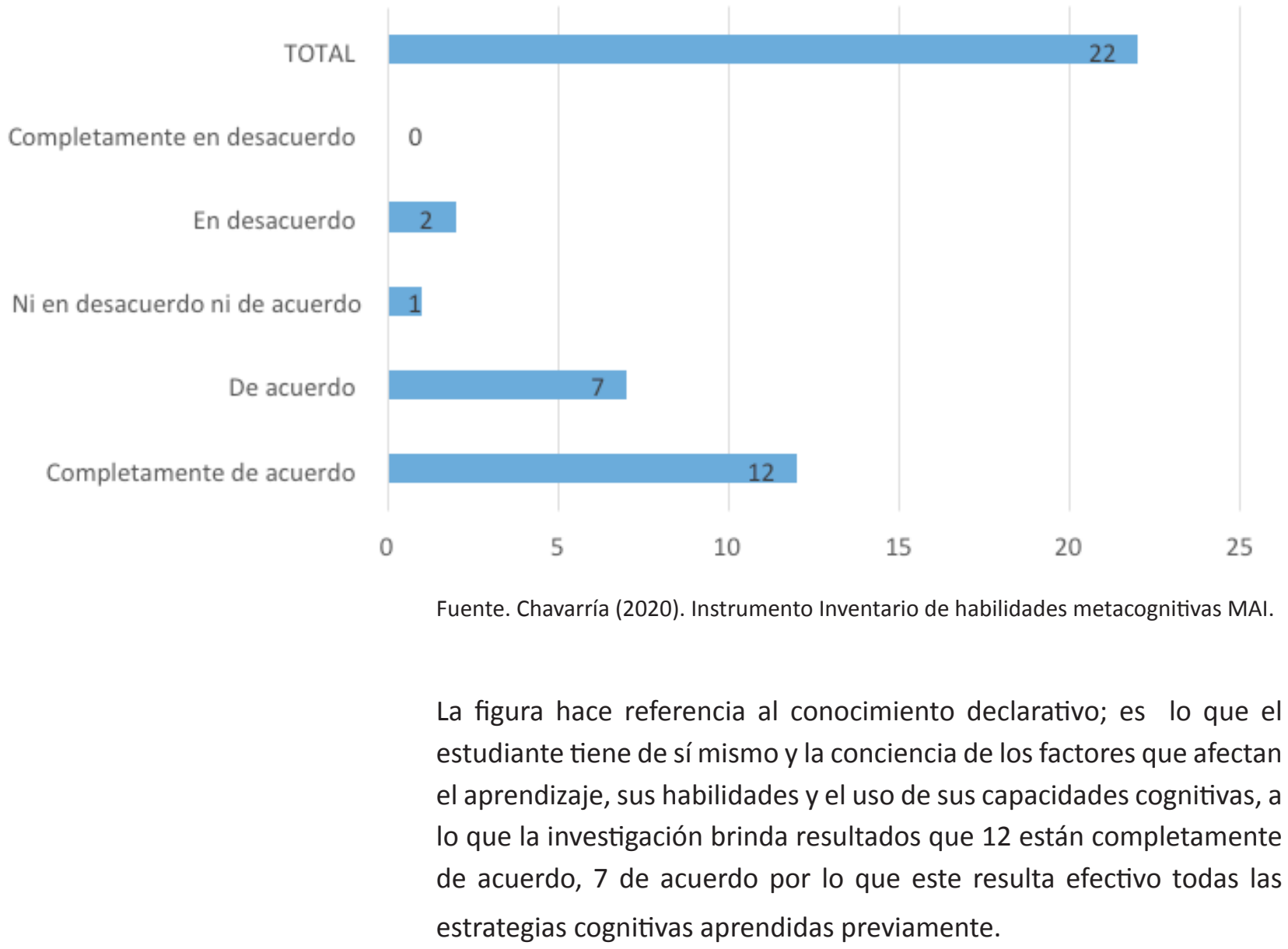


Figura 2. Resultados de la categoría de conocimiento condicional

\section{CONOCIMIENTO CONDICIONAL}

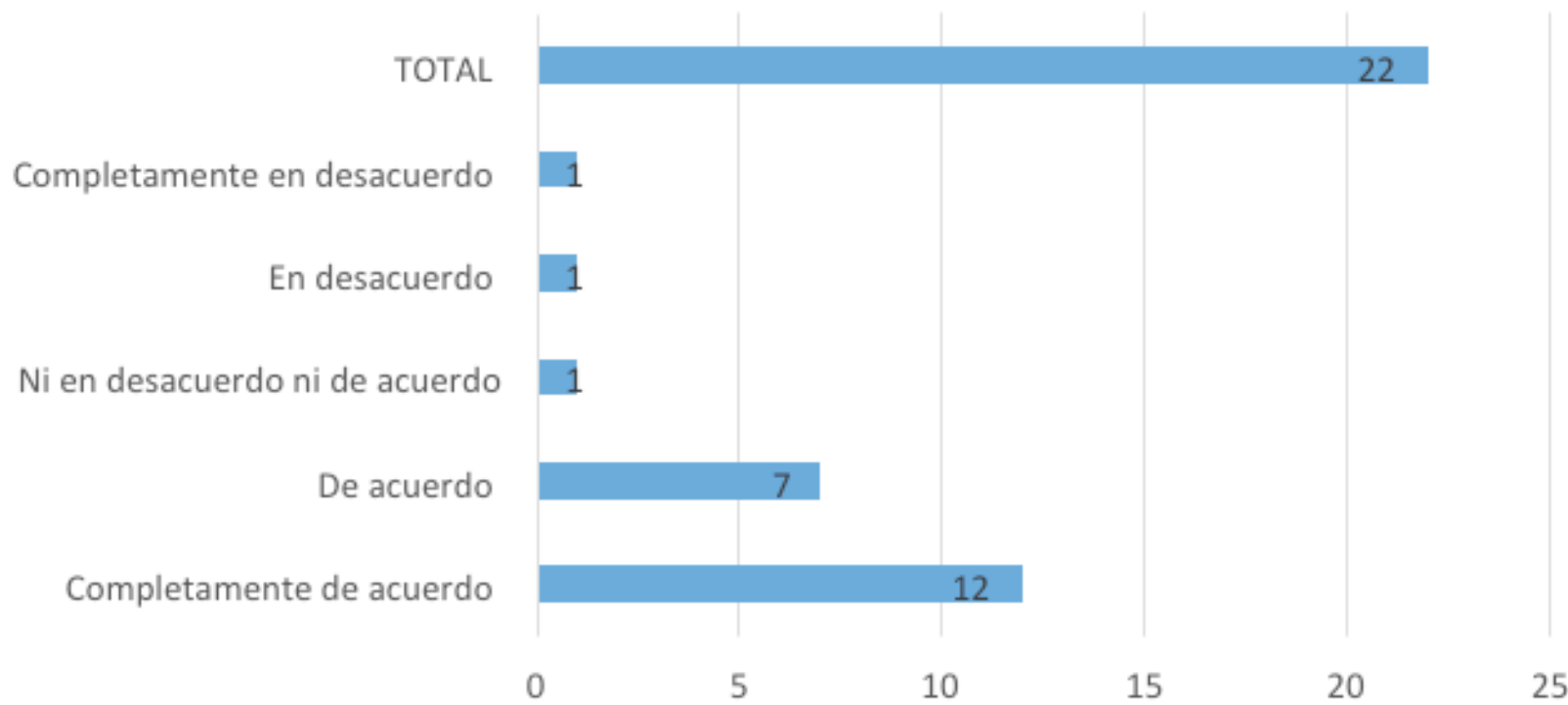

Fuente. Chavarría (2020). Instrumento Inventario de habilidades metacognitivas MAI.

La figura hace referencia al conocimiento condicional que se refiere a cuándo y porque se utilizarán las estrategias de aprendizaje, la investigación evidencia que 19 personas están completamente de acuerdo y de acuerdo en que las utiliza en los procesos de aprendizaje en la educación superior, es importante mencionar que solo 3 están en desacuerdo.

Tabla 1. Sistematización de los datos del cuestionario de funciones ejecutivas

\begin{tabular}{llll}
\hline Funciones ejecutivas & Interpretación & Análisis \\
& Si & No & \\
\hline Planificación & 22 & 0 & $\begin{array}{l}\text { De acuerdo con este resultado se refleja que las personas } \\
\text { entrevistadas el } 100 \% \text { planifican sus actividades académicas. } \\
\text { Anticipación }\end{array}$ \\
& 22 & 0 & $\begin{array}{l}\text { Las } 22 \text { personas están en la capacidad de anticiparse en las } \\
\text { funciones ejecutivas al realizar actividades de aprendizaje } \\
\text { en educación superior. }\end{array}$ \\
\hline
\end{tabular}

Fuente. Chavarría (2020). Cuestionario de funciones ejecutivas. 
Esta tabla muestra que los 22 estudiantes que fue la población total que en las funciones ejecutivas: la planificación y anticipación ellos planifican sus actividades académicas y están en la capacidad de anticiparte en la realización de actividades de aprendizaje en educación superior.

\section{Discusión}

Estos datos indican que las funciones neurocognitivas se van desarrollando a lo largo de los procesos de aprendizaje en la educación superior, tienen mayor diferencia que el conocimiento declarativo y conocimiento condicional; por lo que se compara que estos procesos se potencializan a lo largo del proceso educativo lo cual viene a fortalecer la capacidad de aprendizaje y se concluye que en la realización del análisis de los procesos neurocognitivos que la planificación y la anticipación son las que brindan un dato alto, mientras que la fijación de metas, racionalización, toma de decisiones, multitareas y la flexibilidad, se evidencian al cumplir con los requerimientos del proceso de educación superior según el criterio de la población investigada y la organización, memoria de trabajo, monitorización no son significativas para su proceso de educación superior.

Por lo que se recomienda que las actividades, técnicas, métodos y metodologías de enseñanza a nivel de educación superior deben de propiciar más y mejores aprendizajes, que sean significativos e instrumentales en los estudiantes universitarios y permitirles experiencias y conocimientos sobre su forma de aprender por la vía de las funciones neurocognitivas y la meta-cognición (Barriga, 2010).

\section{Referencias}

Barriga. (2010). Estrategias docentes para un aprendizaje significativo. México: McGrawHill.

Bonilla, G. (2017). Docencia universitaria andragógica . Guatemala : Serviprensa .

Hernández, R., \& Mendoza, C. (2018). Metodología de la investigación. México: McGraw Hill. 
Mayer, R. (2006). Psicología de la Educación. España : Pearson .

Páez, I. (2006). Estrategias de aprendizaje . Revista de Educación Laurus , 260-262.

Rios et al. . (2017). Análisis descriptivo por género de las funciones neurocognitivas en alumos de medicina y nutrición. Ciencias de la Salud, 1-13.

Tobon, et al. (2010). Aprendizaje y evaluación de competencias. México: Pearson.

\section{Sobre la autora}

Es Técnico en Fisioterapia, Técnico en Terapia Ocupacional y Recreativa, Licenciada en Psicología, Especialización en Psicología Forense. Realizó investigaciones previas como la "Rehabilitación neurocognitiva en demencia senil a través de la terapia ocupacional" y "Habilidades neurocognitivas y reminiscencia en las personas institucionalizadas de la tercera edad" y próxima a graduarse en la Maestría en Docencia Universitaria en la Universidad de Guatemala de San Carlos de Guatemala.

\section{Copyright (c) Mariela Dayane Chavarría Pérez}

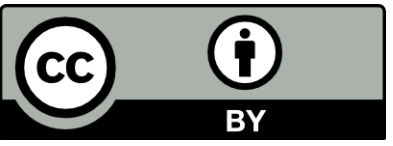

Este texto está protegido por una licencia Creative Commons 4.0.

Usted es libre para compartir, copiar y redistribuir el material en cualquier medio o formato y adaptar el documento, remezclar, transformar y crear a partir del material para cualquier propósito, incluso comercialmente, siempre que cumpla la condición de atribución: usted debe reconocer el crédito de una obra de manera adecuada, proporcionar un enlace a la licencia, e indicar si se han realizado cambios. Puede hacerlo en cualquier forma razonable, pero no de forma tal que sugiera que tiene el apoyo del licenciante o lo recibe por el uso que hace. 